\title{
An Efficient Neighbor Knowledge Based Broadcasting for Mobile Ad Hoc Networks*
}

\author{
Sung-Hee Lee and Young-Bae Ko \\ College of Information and Communication, Ajou University, Suwon, South Korea \\ \{sunghee, youngko\} aajou.ac.kr
}

\begin{abstract}
Although a flooding is the most commonly used method for one-toall communications, it often results in huge traffic in mobile ad hoc networks. Several optimization schemes have been proposed to alleviate this problem. However, none of them offers a complete solution and has their own limitations, such as, lack of reliability, high latency or excessive control packet overhead. In this paper, we propose the FONIAH (Flooding based on Onehop Neighbor Information and Adaptive Holding) scheme to overcome these limitations.
\end{abstract}

\section{Introduction}

In mobile ad hoc networks (MANET), a flooding is the simplest method to disseminate a certain message from a single node to everyone in a network. With the simplest blind flooding, any node is required to rebroadcast a receiving packet to its neighbors unless it has already received a duplicate packet. Although flooding is simple and easy to implement, it often results in a large number of network wide redundant packets. This situation is also referred in literature as the broadcast storm [1].

Many routing protocols and applications in MANET rely on flooding (or its variants) for a route discovery or data dissemination. Previous studies show that the reliability achieved by employing the blind flooding comes at the cost of overall network performance degradation [2]. There are several disadvantages associated with the blind flooding such as excessive redundant packets, heavy contentions and collisions [1]. To alleviate these problems, a considerable number of protocols have been presented with the same good of developing more efficient broadcasting schemes. In [3], the authors categorized these protocols into four families: Simple flooding (= blind flooding), Probability based flooding, Area based flooding, and Neighbor knowledge based flooding. Probability based flooding is defined to use a certain predetermined probability to decide whether each node rebroadcasts a receiving packet or not. Area based flooding requires a node to evaluate the additional

\footnotetext{
This research is supported by the Ubiquitous Autonomic Computing and Network Project, the $21^{\text {st }}$ Century Frontier R\&D Program, and the ITRC (Information Technology Research Center) support program supervised by the IITA(Institute of Information Technology Assessment), the Ministry of Information and Communication(MIC) in Korea.
} 
area covered by its rebroadcasting. If this additional coverage is less than some threshold value, the node will give up its rebroadcasting. Neighbor knowledge based flooding utilizes two hop neighbor information obtained via HELLO packets to reduce redundant broadcasting. Thus, this Neighbor knowledge method may allow a node receiving a packet to do rebroadcast, only when its rebroacasting results in any additional neighbors to be reached. As the authors in [3] argued, all these flooding approaches have their own strengths and weaknesses in terms of algorithm complexity, additional overhead, and so on.

In this paper, we take some hybrid approach combining Neighbor knowledge based flooding and Area based flooding, and propose a new algorithm called as FONIAH (Flooding based on One-hop Neighbor Information and Adaptive Holding). The proposed FONIAH utilizes knowledge of 1-hop neighbors and the concept of "adaptive holding time," which is motivated from one of the area-based flooding protocol named GeoFlood [4]. In [4], the concept of "Packet holding time" is defined to allow a node receiving a packet to hold it for some amount of time before rebroadcasting. This holding time is incremental as the distance from the sender to the receiver decreases, so a node closer to the sender will become waiting longer. Although this concept can be useful to rapidly enlarge a coverage area, it may cause a high latency problem in a sparse network. Through the simulation study with ns-2, we have shown that the proposed scheme provides better efficiency without harming delivery effectiveness, compared to the existing schemes of either purely Neighbor knowledge based or Area based approaches.

\section{The Proposed Scheme: FONIAH}

We assume that each node knows its own geographical location, and periodically sends HELLO packets containing its location information. Upon receiving a HELLO packet, a node updates its neighbor table. Consequently, every node becomes to know geographical locations of all neighbors within one hop transmission radius.

In the proposed scheme, at first, a broadcasting initiator node looks up the neighbor table to find a farthest neighbor within one hop radius, and then calculates the distance from itself to the farthest neighbor. This distance is referred to as Maximum Distance $\left(d_{\max }\right)$, and it is embedded in the broadcast packet with the location of the sender node. $d_{\max }$ value is utilized to calculate a packet holding time at the receiver node. This embedded information is updated by the successive next hop nodes.

On receiving a broadcast packet, a node waits for a certain holding time which is inversely proportional to the distance from the sender to itself. When a holding time expires, a node updates the value of $d_{\max }$ and the location of the sender node in the packet, and then rebroadcasts it. We propose a simple linear function to obtain a packet holding time as follows:

$$
H T_{\text {cur }}=H T_{\max }-\frac{d_{c u r} \times H T_{\max }}{d_{\max }}
$$

$H T_{\max }$ is the predetermined, constant maximum holding time. $d_{c u r}$ is the distance from the previous hop node to the current node. It is calculated from the location of the previous hop node embedded in the receiving packet. $d_{\max }$ is also embedded in the packet. A current holding time $H T_{c u r}$ is a function of $d_{c u r}$ and $d_{\max }$. As a result, a 
current holding time is inversely proportional to the distance between the sender and receiver node. That is, the farthest node from the sender node rebroadcasts earlier than any others. It helps to enlarge a coverage area rapidly because outer nodes cover more new network space than inner nodes.

Since the maximum distance is fixed to the maximum transmission range in GeoFlood, a high latency problem occurs in a sparse network as we mentioned before. However, in our scheme, this value is not fixed and it is just supplied by the previous hop node via the packet. Therefore, unlike GeoFlood, the farthest neighbor always has zero holding time wherever it is placed. We refer this mechanism to the Adaptive Holding Time.

When a node receives a new broadcasting packet, it creates a Mark Table per packet. This mark table represents which neighbors have yet received the packet. Waiting for the packet holding time to expire, a node decides whether to rebroadcast a receiving packet or not, based on the following procedure. When a node receives a redundant packet during the holding time, it estimates which of its neighboring node has also received that packet. A receiver node calculates the distance from the packet sender to each own neighbor, and compares it with $d_{\max }$ value embedded in the packet. If this distance is shorter or equal to $d_{\max }$, this neighbor supposes to receive that packet. This process is repeated when a redundant packet arrives. Finally, before a holding time expires, if all neighbors in the mark table are marked - that is all neighbors have already received the packet, the deferred packet is dropped. Otherwise, that packet is rebroadcasted. A mark table is also removed when a holding time expires.

\section{Performance Evaluation}

We performed a simulation study using ns-2. In our simulation model, nodes are randomly deployed in $1000 \mathrm{~m} \times 1000 \mathrm{~m}$ square area and move according to a random movement pattern at maximum speed of $5 \mathrm{~m} / \mathrm{s}$ without any pause. A broadcast packet is initiated at every 1 second from a randomly selected node with fixed-size payload of 64 bytes which is equal to the size of typical RREQ message in AODV. We use the IEEE 802.11 MAC with a bandwidth of $2 \mathrm{Mbps}$ and a maximum transmission range of $250 \mathrm{~m}$. In FONIAH, each node sends a HELLO packet at every 5 seconds. Total simulation time is 300 seconds and we repeat each scenario five times with different random seed numbers. We select $50 \mathrm{~ms}$ of the maximum holding time for GeoFlood and FONIAH.

We evaluate our scheme using the following metrics. Packet delivery ratio is defined as a ratio of the number of nodes receiving the flooded packet to the number of nodes in the network. Total packet overhead is defined as the total size of all transmitted data and control packets. This metric reflects the total routing load involved in flooding and hence protocol efficiency. The end-to-end latency is measured as a difference in time from when the source node initiates flooding to when other nodes receives it. We report the average latency over all nodes.

Fig. 1(a) presents the delivery ratio as a function of the number of nodes. When the number of nodes is 20 , the delivery ratio of three schemes is dropped up to $83 \%$, because the network density is too sparse to support the network connectivity. In 
Fig. 1(b), we observe that the total packet overhead in FONIAH is smaller than GeoFlood and Blind Flooding. The difference of the packet overhead becomes larger as the number of nodes increases. In Fig. 1(c), the average latency of our scheme is always smaller than GeoFlood. It represents that the adaptive holding time mechanism in FONIAH is more efficient than GeoFlood in the end-to-end packet latency. Especially, the difference between GeoFlood and FONIAH is larger in the small number of nodes, since our scheme alleviates the high-latency problem of GeoFlood in a sparse network. Even though the latency is still longer than simplest blind flooding, a reduced packet overhead is helpful to improve the network performance.

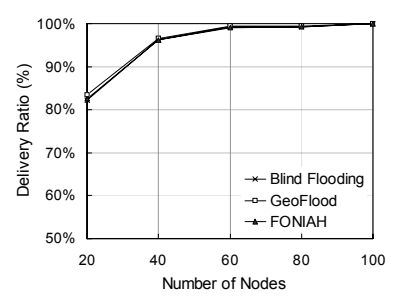

(a) Delivery Ratio

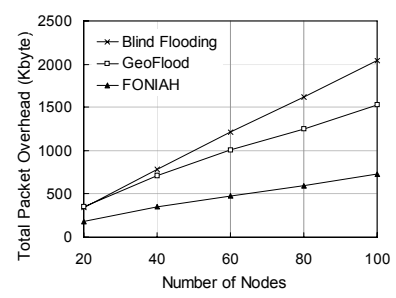

(b) Total Packet Overhead

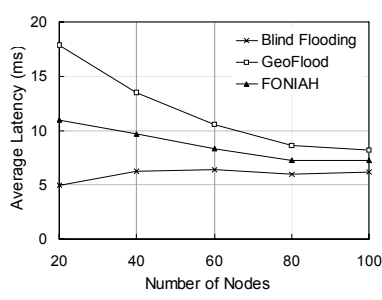

(c) Average Latency

Fig. 1. Simulation results with respect to the number of nodes

\section{Conclusion}

The proposed FONIAH (Flooding based on One-hop Neighbor Information and Adaptive Holding) scheme provides a more efficient solution to alleviate the problem caused by blind flooding. Our scheme combines both Neighbor knowledge based flooding and Area based flooding to overcome the limitations in each approach. Simulation results shows that our scheme has low packet overhead, reasonable latency and high reliability. Future works would include some complexity analysis of the proposed algorithm and the study for further reducing its end-to-end latency.

\section{References}

1. S.-Y. Ni, Y.-C. Tseng, Y.-S. Chen, and J.-P. Sheu, "The broadcast storm problem in a mobile ad hoc network," in Proc. of MobiCom, pages 151.162, 1999.

2. J. M. McCune, E. Shi, A. Perrig and M. K. Reiter, "Detection of Denial-of-Message Attacks on Sensor Network Broadcasts," in Proceedings of IEEE Symposium on Security and Privacy, pp. 64-78, 2005.

3. B. Williams, T. Camp "Comparison of Broadcasting Techniques for Mobile Ad Hoc Networks," in Proc. of the third ACM international symposium on Mobile ad hoc networking \& computiong, pp.194-205, June, 2002

4. J. Arango, M. Degermark, A Efrat, S. Pink "An Efficient Flooding Algorithm for Mobile Ad-hoc Networks", in Proc. of WiOpt, 2004 